\title{
'All these allied health professionals and you're not really sure when you use them': Insights from Australian International Medical Graduates on working with Allied Health.
}

\begin{abstract}
:
Objective: This paper presents the findings of research which explored how International Medical Graduates (IMGs) understand and integrate with the allied health system in relation to multidisciplinary care.
\end{abstract}

Methods: An open-ended, exploratory qualitative design comprised of thirty ( $\mathrm{n}=30$ ) open-ended, in-depth interviews with IMGs employed in a public hospital in Queensland, Australia.

Results: Many IMGs have no experience with allied health support in their country of origin. Multidisciplinary collaboration is a new concept for IMGs integrating into the Australia health care system. Learning about the allied health system, including how to work effectively within the context of the multidisciplinary team, is an important topic that needs to be addressed as a matter of priority.

Conclusions: There is a strong need to focus on improving strategies for integrating IMGs into the allied health system. In particular, IMGs require information to help them understand the roles and referral processes associated with interfacing with the allied health system. 


\section{'All these allied health professionals and you're not really sure when you use them': Insights from Australian International Medical Graduates on working with Allied Health.}

In Australia, an international medical graduate (IMG) is defined as a doctor who has obtained a primary medical qualification in a country other than Australia or New Zealand. ${ }^{1}$ Because of problems associated with physician shortages, many countries (including Australia) are increasingly forced to rely on IMGs to meet medical workforce

needs. ${ }^{2,3,4}$ By way of example, IMGs now represent 25 percent of the total workforce of Australian physicians. ${ }^{5,6}$

\section{WHAT IS KNOWN ABOUT THE TOPIC}

Although work in this area is in its infancy, there is now an increasing literature on professional issues associated with integration of IMGs. ${ }^{1,2,3,4,5,6,15,16,17,18}$ However, to date, there is no research available on the important topic of how IMGs understand and integrate with the allied health system in relation to multidisciplinary care. This article provides seminal research in this area by presenting insights from Australian IMGs that indicate that they have scant prior experience with allied health in their country of origin and are eager to learn about this holistic and multidisciplinary approach to patient care.

Whilst applauding O’Kane and Lowe's ${ }^{7}$ comprehensive definition, the term 'allied health' for this paper resonates with the narrower definition of Boyce ${ }^{8}$ that is common in many institutional settings comprising core professions of social work, psychology, dietetics, speech pathology, occupational therapy and physiotherapy. 
As the IMGs interviewed for the research were all employed at a public district hospital in Queensland, the focus for the following discussion is on public hospital-based IMGs and allied health professionals. This resonates with the general employment situation in relation to Australian allied health professionals who are predominantly employed in the public sector, especially in the public hospital system. ${ }^{9}$

\section{THE SETTING}

The hospital is a 140-bed general hospital that provides emergency, medicine, surgery, obstetrics and gynaecology, paediatrics and mental health services. The hospital is staffed by senior full-time medical staff and 'junior' or trainee staff. There is a standard complement of allied health staff, including physiotherapists, occupational therapists, speech pathologists and social workers, deployed in the inpatient wards and emergency

department. There are well-developed systems of multidisciplinary care involving medicine, nursing and allied health, which include discharge planning, aged care assessment and general social and psychological support.

\section{THE RESEARCH}

The study, funded by an Industry Grant, represents collaboration between Central Queensland University ('University') and the hospital. The study is part of a broad program of research exploring the integration of IMGs into the Australian health system, and the specific aim of this research project was to examine the experience of integration from the IMGs’ perspective. 
The participants were enrolled through a University based project officer independent of the hospital. The project officer was given a list of doctors who had been employed at the hospital, along with their telephone numbers. This information was obtained from a hospital representative who had notified potential participants about the details of the study by email and allowed any IMGs to opt out of further follow-up if so elected. The participants were consecutively enrolled from this list, through an initial telephone call or email contact, followed by the project officer providing written Project Descriptions of the project and an invitation for voluntary participation in the research. At this stage signed consent forms from the participants were collected and enrolment occurred. There was no screening of participants. Prior to interviewing, participants were again informed of their ethical rights (e.g. informed consent, confidentiality, right to withdraw). The University ethics committee and the Queensland Health Department Human Research Ethics Committee approved the study.

\section{Participants}

Thirty (n=30) IMGs who were presently working at the hospital were interviewed. The primary medical degree held by the IMGs were from a range of countries: India ( $\mathrm{n}=11)$;

Sri Lanka ( $\mathrm{n}=4)$; Iran ( $\mathrm{n}=3)$; South Africa $(\mathrm{n}=2)$; Sudan $(\mathrm{n}=2)$; Pakistan $(\mathrm{n}=1)$; Caribbean ( $\mathrm{n}=1)$; Russia ( $\mathrm{n}=1)$; Philippines ( $\mathrm{n}=1)$; Indonesia ( $\mathrm{n}=1)$; Egypt $(\mathrm{n}=1)$; Serbia $(\mathrm{n}=1)$; Afghanistan (n=1). The majority of IMGs (n=20; $66.6 \%)$ came directly from their country of origin to practice in Australia. The IMGs who spent time in other countries prior to coming to Australia predominately went to the United Kingdom ( $\mathrm{n}=5 ; 16.6 \%)$ with others spending time in the health system in Tehran $(\mathrm{n}=1)$, New Zealand $(\mathrm{n}=2)$ 
Oman and Fiji (n=1) and Pakistan (n=1). The length of time that the IMGs had been in Australia ranged from two to 17 years (2008 n=4; $2007 n=3 ; 2006 n=2 ; 2005 n=6 ; 2004$ n=3, $2002 n=1,2001 n=1,1999 n=1 ; 1998 n=1,1997 n=1 ; 1995 n=1,1994 n=1,1993$ $\mathrm{n}=1$, not available $\mathrm{n}=4$ ).

Because the participants were enrolled from a small, identifiable group at the hospital the informed consent procedures gave a strict commitment to confidentiality and a guarantee that no further identifying information would be presented or published with the findings. Hence, further demographic description will not be provided to protect the identity of the participants.

\section{Research Design}

An open-ended, exploratory qualitative design was utilised for the study. Qualitative research is used to evaluate programs in health care to provide insights on quality and effectiveness and to assist in program improvement. ${ }^{10,11}$ Such a qualitative approach is particularly appropriate where little is known about an issue $e^{12,13}$ and so is well suited to a study that explores the experience of integration for IMGs where there is scant research literature available.

\section{Interviews}

The exploration of the IMGs' experience with integrating into the Australian health system was conducted through an iterative, qualitative research methodology using openended interviews conducted at the time and location of each participant's choice. The interviews were conducted by a psychosocial researcher with a background in cross- 
cultural research employed by the University and thus independent of the hospital. The interviews were conducted by speaker-phone.

The IMGs were encouraged to talk about their experience as a doctor prior to, during and following their integration into the Australian health system. The interviews were openended and driven by an active listening to the insights of the IMGs. According to the iterative principle of qualitative research issues posited as important in early interviews were explored in subsequent interviews. The issue with regards allied health was posited by the IMGs and explored in all of the interviews. The line of questioning included the techniques of probing, paraphrasing and silence to explore each participant's experience. ${ }^{14}$ The interviews lasted for approximately one hour and were audio-recorded. The interviews were transcribed verbatim by a research assistant independent of the hospital.

Analysis

As descriptive qualitative research the analysis was driven by a commitment to representing the insights and perspective of the participants. The task of the descriptive qualitative research analysis is not to mediate the findings but rather to present a description of the emic or insider insights that resonates with the exact words of the participants. The language texts were then entered into the QSR NUD*IST (N5 1995) computer program and analysed thematically. All of the participants' comments were coded into 'free nodes' which are category files that have not been pre-organised but are 'freely' created from the data. The list of codes was then transported to a Word Computer Program (Word 97) and organized under thematic headings. The coding was established 
by an experienced qualitative researcher and completed by the project officer who has extensive experience with coding qualitative data. There was complete agreement on the coding and emergent themes. Of the 201 free nodes created from the transcriptions the ones directly related to the topic of the IMGs' experience with the allied health system are presented in this article.

\section{FINDINGS}

\section{Allied health - an important IMG health systems issue}

Participants posited the difficulties associated with functioning effectively in the health care system as a core integration concern that was seen as more important than overcoming any differences in clinical knowledge, for example:

[Sudan] It's challenging for us, you know. I don't know, but ah, to me this is one of, the, the most difficult parts... to get through the... Australian system.

Understanding the role of and how to interface with allied health professionals was noted as a major systems issue:

[India] I've always found it difficult to make maximal use of the resources that's available. Ah, you have all these allied health professionals and you're not really sure when you use them, or when you don't use them.

On a positive note, the IMGs were enthusiastic to learn about the Australian allied health system and embraced a perspective of ongoing learning in this area, for example: 
[Afghanistan] Yeah, still lots of learning many ways to help patients and it’s good to involve allied health in the patient care and I'm learning how so many ways, how they help you know like social workers, occupational therapists, physios even when the patient goes you know home still they can get services.

\section{No allied health available in country of origin}

At the centre of the problem of IMGs lack of knowledge about the allied health care system is the fact that most come from countries of origin that have scant, if any, allied health professionals. Participants from countries as diverse as Afghanistan, India, Sri Lanka and Pakistan made statements such as,

[Sri Lanka] Ah, yeah, in my country usually we don’t have this kind of integrated system.

Participants indicated that for some countries such as India, allied health is in its infancy and is 'not mainstream' and mostly in the private health care system.

As can be seen by the following statement, the IMG can bring a professional perspective that does not look further than the clinical encounter. The doctor is not trained to even consider allied health care issues.

[Iran] I had no experience of that in my country [laughs] so, it’s something totally new. So it's really just doctor treating , the patient's problem and we don't, this is not good, but I think we in my country we don't care what happens next.

The health care system of the country of origin of many of IMGs interviewed was described as medico-centric, with doctors not having the time or resources to explore 
holistic care issues with patients, for example:

[India] If you [patient] go to the hospital there, it's basically to see the doctor. And just don't want an opinion from anyone else. The patients, they want the doctor to do most of the things.

[Caribbean] They [doctors] don't have that five minutes extra, or a minute extra to sit down with that patient and talk to them.

The IMGs made very positive comments about the Australian allied health system and expressed appreciation for the opportunity to engage with patients in collaboration with such a system, for example:

[Iran] So I really do like what happening here because even with a patient which has a fracture, if they think that there are some stairs that the patient needs to go up stairs they won't send the patient home. Ah, I really like this system here and I had no idea that something like this can happen.

Different cultures - in country of origin it is usually the family that does the work of allied health

The lack of integration of allied health in many of the countries of origin for IMGs is driven by a cultural imperative that in those countries the family is seen as the key provider of holistic care, for example,

[India] Ah, the families do it all in India, we don't usually ask them and where do they come from, because we know that they got a home and they live with their family. 
As the issue is deeply embedded in a cultural perspective, the IMGs emphasised the importance of gaining information on cultural issues in order to be able to understand how to relate to patients appropriate to the culture of the Australian health system, for example,

[Philippines] But it depends on the culture. Yes I think that is very important, knowing the culture.

\section{Multidisciplinary collaboration a new concept for IMGs}

Learning to participate in multidisciplinary team collaboration is noted by the IMGs interviewed to be a new, unfamiliar and important aspect of learning to integrate into the Australian health system. When discussing the issue of allied health the IMGs highlighted the fact that multidisciplinary team work was a very different modus operandi than they were used to in their country of origin, for example,

[Sudan] So when I came here I found out we work as you know, as more like a team. Overseas countries not have this system, this organised system. Especially how to work as a team.

\section{A topic that IMGs need to learn about}

In summary, learning about the allied health system, including how to operate within the context of the multidisciplinary team, is considered to be an important topic that needs to be addressed in integrating IMGs into the Australian health care system. The participants made direct calls for assistance in understanding the allied health system, for example: 
[India] And it would be extremely useful for us to understand what the rules of allied health fields are.

[Sudan] What the role of OT, what the role of physiotherapy, what the role of the social worker... So you can’t catch all this information, you know, by yourself.

They acknowledged that, even after working in the system for some time, their knowledge of this area is still not complete. :

\section{Strategies for educating IMGs about allied health}

The participants spoke in depth about strategies they believed would assist them to learn about the allied health system and functioning in the multidisciplinary team. Most

importantly, the participants indicated that they should be informed about this system, and that the leadership for this should come from the allied health professionals,

[Sudan] I think it’s important for allied health to take responsibility for telling us about it. Especially the new doctors. They usually assume that you know the roles.

Some IMGs indicated that it was through the support of allied health professionals that they were helped to understand the allied health system.

Essentially the IMGs require information to help them understand the roles and referral processes associated with interfacing with allied health. A few of the IMGs had the advantage of experience in other countries that have an allied health system (e.g. United Kingdom; Oman; New Zealand) which provided them with basic information, including 
about appropriate processes. It was also suggested that IMGs would benefit from access to information about the system before they come to Australia.

It was noted that the process of interfacing with allied health is built into the orientation program for newly arrived IMGs. This orientation needs to cover the full range of allied health support along the continuum of treatment from the point of diagnosis to palliative care. ,

There was some concern that the topic was too extensive to be covered effectively in the short orientation program offered to IMGs at the hospital, as one participant explained:

[Egypt] I got orientation program, it was a very good one, but only a day. But guess we need more than one orientation program.

There was some difference of opinion about the best way to give information and to orient the IMGs to work with the allied health system. Some IMGs indicated that providing lectures/workshops on the topic would not be helpful because the learning focused on culturally complex practice-based concerns.

Others indicated that workshops and seminars, not only during orientation but also as a form of continuing professional education, would be useful.

There was, however, strong agreement that understanding the role of allied health was something that needed to be learned at the 'coalface' during the actual day-to-day process of patient care. Interfacing with allied health was seen as a practice that needed to be 'learned at the coalface' and understood by 'personal experience'. 
There were specific times during the daily care of patients that the IMGs considered most productive in terms of learning about the interface of allied health. These time included ward rounds, hand-over, patient discharge and multidisciplinary meetings. In terms of learning from the 'coalface' the IMGs indicated that other doctors, both senior and peerlevel, can be an important source of information, guidance and support in relation to knowing how to appropriately interface with allied health.

\section{DISCUSSION}

In recent decades there has been an increase in the inter-country mobility of physicians around the world. ${ }^{15}$ Doctors leaving their country of origin face a range of challenges in order to practice medicine in a different geographical and cultural setting.

As detailed in Diagram 1, the IMGs in this study indicated that learning about the health system is the major challenge to integration into Australian health practice. Canadian research on IMGs indicates that understanding the health care system is rated as the most

important need of IMGs. ${ }^{16}$ Similarly, Curran and associates ${ }^{17}$ report that IMGs entering employment may experience difficulty understanding how medicine is organised in countries new to them. IMGs are identified as a group that has a poor familiarity with the Australian health system, especially when compared to local graduates. ${ }^{1,18}$

\section{WHAT THIS PAPER ADDS}

Although the literature acknowledges the need for IMGs to understand the health system, to date, there is no research specifically in relation to IMGs' understanding of allied health and multidisciplinary care. The present findings make an important contribution 
to the literature by highlighting the need to focus on integrating IMGs into the allied health system. The findings presented in this paper indicate that, for the range of reasons summarized in Diagram 1, the IMGs' understanding of the allied health system is limited. Thus, understanding how to operate in an environment of multidisciplinary care and integrate with the allied health professionals is a key systems issue that IMGs seek to understand. This is an important professional issue, for as Dubouloz and associates ${ }^{20}$ indicate, collaborative work among health care professions is the key to quality patient/client care and inter-professional care is the way to the future. There is a need for health professionals to be able to work across disciplines to ensure effective, competent, and culturally sensitive health care delivery. ${ }^{25}$ The findings are further evidence of the importance of and need for inter-professional education already noted in the literature. $^{21,23}$

The holistic perspective and multidisciplinary team approach of allied health, although different to the medico-centric system of their country of origin, is valued and IMGs are highly motivated to learn about this different modus operandi of patient and family care. The IMGs understanding of their need to undertake learning and improvement in this regard is, according to Wilcock and associates ${ }^{24}$, a key factor that will foster the promotion of continuing interprofessional development in the workplace and ultimately enhance patient outcomes.

\section{INSERT DIAGRAM 1 HERE}


The IMGs considered that allied health professionals are well placed for leadership in driving educational opportunities for IMGs to learn about how doctors can effectively interface with allied health. As detailed in Diagram 2, the IMGs outlined a range of educational strategies and points of learning that can be used to optimise their understanding in this area. As indicated, experience working in the system is likely to be more effective than purely instructional orientation. This insight in relation to interprofessional learning is affirmed by Conway ${ }^{22}$ who indicates that clinical practice provides the stimulus for practitioners to recognize best practice and, if necessary, enhance and modify existing practice. Educational sessions have also been identified as an effective strategy enabling interprofessional learning. ${ }^{26}$ Educational initiatives will be significantly assisted by an awareness of the unfamiliarity of IMGs with the systems of multidisciplinary care and the need and methods of negotiating with allied health professionals.

INSERT DIAGRAM 2 HERE

\section{IMPLICATIONS FOR PRACTITIONERS}

As Boyce ${ }^{8}$ indicates, the public hospital, particularly the acute care general hospital, is now seen as a 'leadership practice centre for allied health professions'. The present findings indicate that leadership should extend to assistance with IMG learning and ongoing professional education about the allied health system. The IMGs in this study called for assistance in understanding the allied health system, a process they considered 
difficult to do on their own. This need for IMGs to receive more attention and assistance than local medical graduates is reported in the literature. ${ }^{1,19}$ The strong recommendation is that it is most appropriate for allied health professionals in the hospital to take such leadership and such efforts will be met by a readiness to learn on the part of the IMGs.

\section{LIMITATIONS}

Further work is required to explore this issue over a broader geographical area and taking into account the perspectives and experiences of allied health professionals who interface with IMGs.

\section{CONCLUSION}

The insights generously provided by Australian IMGs from a range of different countries of origin clearly indicate the need to prioritise knowledge about the holistic and multidisciplinary team approach of the allied health system in strategies for IMG integration. These new insights into the importance of allied health leadership in professional health education are offered in the hope of not only meeting IMGs professional education needs, but also ensuring that the benefits of allied health care are well understood and acted upon by all doctors working in the Australian health care system.

Acknowledgement: The author would like to acknowledge Ms Stasia Kail-Buckley, Mrs 
Elaine Phillips, Ms Amy Carson and Ms Mary Anne Patton for their contribution to the study and Ms Emma Phillips for proof reading the manuscript. The authors would also like to thank Metro South Health District, Queensland Health, for the funding for the study. 


\section{REFERENCES}

1. Sullivan E, Wilcock S, Ardzejewska K, Slaytor E. A pre-employment programme for overseas-trained doctors entering the Australian workforce, 1997-99. Med Educ 2002; 36: 614-621.

2. Heal C, Jacobs H. A peer support program for international medical graduates. Aust Fam Physician 2005; 34, 4: 277-278.

3. McGrath B. Integration of overseas-trained doctors into the Australian medical workforce. Med J Aust 2004; 161, 11/12: 640-642.

4. Mullan F. The metrics of the physician brain drain. $N$ Engl J Med 2005; 353: 18101818.

5. Pilotto L, Duncan G, Anderson-Wurf J. Issues for clinicians training international medical graduates: a systematic review. Med J Aust 2007; 187, 4: 225-228.

6. Pincock S. Overseas doctors work longer hours, says Australian study. BMJ 2007; 335, 7614: 274.

7. O’Kane A, Lowe S. Clinical Allied Health Professions - A Method of Classification Developed from Common Usage of the Term ‘Allied Health' - as Applicable to Rural and Remote Australia. Canberra: Services for Rural and Remote Allied Health (SARRAH), 2003.

8. Boyce R. Emerging from the shadow of medicine: allied health as a 'profession community’ subculture. Health Sociology Review 2006; 15, 5: 520-534.

9. Australian Health Workforce Advisory Committee (AHWAC). The Australian Allied Health Workforce: An Overview of Workforce Planning Issues. Sydney: AHWAC: 2004. 
10. Holloway I. A-Z of Qualitative Research in Healthcare. 2nd edn. Oxford: Blackwell Publishing, 2008.

11. Patton M. Qualitative Research and Evaluation Methods. $3^{\text {rd }}$ rev edn. Thousand Oaks: Sage, 2002.

12. Krathwohl D. Methods of educational and social science research: An integrated approach. New York: Longman, 1993.

13. Polit D, Hungler B. Nursing research: principles and methods. $5^{\text {th }}$ edn. Philadelphia: Lippincott, 1995.

14. Gaskill D, Henderson A, Fraser M. Exploring the everyday world of the patient in isolation. Oncol Nurs Forum 1997; 24, 4: 695 - 700.

15. Eckhert NL. The global pipeline: Too narrow, too wide or just right. Med Educ 2002; 36, 1: 606-613.

16. Zulla, R, Baerlocher M, Verma S. International medical graduates (IMGs) needs assessment study: comparison between current IMG trainees and program directors. BMC Med Educ 2008; 8: 42.

17. Curran V, Hollett A, Hann S, Bradbury C. A qualitative study of the international medical graduate and the orientation process. Canadian Journal of Rural Medicine 2008; 13, 4: 163-9.

18. Rolfe I, Andrew J, Pearson S, Hensley M, et al. Clinical Competence of interns, Med Educ 1995; 29: 225-230.

19. Postgraduate Medical Council of New South Wales (PMCNSW). Directors of Clinical Training Survey, Sydney. Postgraduate Medical Council: New South Wales, 1997. 
20. Dubouloz C, Savard J, Burnett D, Guitard P. An interprofessional rehabilitation university clinic in primary healthcare: a collaborative learning model for physical therapist students in a clinical placement. Journal of Physical Therapy Education 2010; 24, 1:19-24.

21. Swisher L,Woodward L, Quillen W, Monroe A. Centralized and decentralized organizational models of interprofessional education for physical therapist and medical student. Journal of Physical Therapy Education 2010; 24,1,:12-8.

22. Conway J. Implementing interprofessional learning in clinical education: findings from a utility-led evaluation. Contemporary Nurse 2009; 32, 1-2 :187-200.

23. Goldman J, Zwarenstein M, Bhattacharyya O, Reeves S. Improving the clarity of the interprofessional field: implications for research and continuing interprofessional education. Journal of Continuing Education in the Health Professions 2009; 29,3:151-6.

24. Wilcock P, Janes G, Chambers A. Health care improvement and continuing interprofessional education: continuing interprofessional development to improve patient outcomes. Journal of Continuing Education in the Health Professions 2009; 29,2:84-90.

25. Pecukonis E, Doyle O, Bliss D. Reducing barriers to interprofessional training: promoting interprofessional cultural competence. Journal of Interprofessional Care 2008; 22,4:417-28.

26. Mann K, Sargeant J, Hill T. Knowledge translation in interprofessional education: what difference does interprofessional education make to practice? Learning in Health \& Social Care 2009; 8,3:154-164. 
Understanding Australian health system issues is the major challenge for IMGs

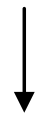

Allied Health care system is core unknown systems issue

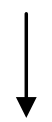

Reasons for IMG lack of understanding of allied health system:

- No or scant allied health system in IMG country of origin

- Not trained to focus on allied health issues

- Exclusive biomedical focus

- Doctor-centric health care system

- No time to work holistically with patients

- Cultural expectation that family does the work of allied health

- No experience with working in multidisciplinary team (MDT) which is a new professional modus operandi

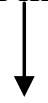

IMGs value and are keen to learn about allied health system

Diagram 1: Background factors on IMGs strong need to understand the Australian allied health system. 


\section{Multi-level Educational Strategy}

- Allied Health leadership

- Build into orientation program

- Needs to be ongoing professional education

- Information giving

- Lectures and workshops (valuable for some)

- Learning at the coalface:

o Direct interface with Allied Health professionals

o Ward rounds

o Hand overs

o Patient discharge

o Multi-disciplinary meetings

o Learn from other doctors

o Some IMG bring experience in Allied Health from work in other Western countries

Diagram 2: Educational strategies needed to inform IMGs of Allied Health system of care. 